\title{
Accelerated partial breast irradiation in an Asian population: dosimetric findings and preliminary results of a multicatheter interstitial program
}

This article was published in the following Dove Press journal:

OncoTargets and Therapy

8 September 2016

Number of times this article has been viewed

\author{
Yaling Vicky Koh' \\ Poh Wee Tan' \\ Shaik Ahmad Buhari ${ }^{2}$ \\ Philip lau ${ }^{2}$ \\ Ching Wan Chan ${ }^{2}$ \\ Liang Shen ${ }^{3}$ \\ Sing Huang $\operatorname{Tan}^{4}$ \\ Johann I-Hsiung Tang' \\ 'Department of Radiation Oncology, \\ National University Cancer Institute \\ Singapore, ${ }^{2}$ Department of Surgery, \\ National University Hospital, \\ ${ }^{3}$ Department of Medicine, Biostatistics \\ Unit, National University of Singapore, \\ ${ }^{4}$ Department of Medical Oncology, \\ National University Cancer Institute \\ Singapore, Singapore
}

Introduction: Accelerated partial breast irradiation (APBI) using the multicatheter method has excellent cosmesis and low rates of long-term toxicity. However, there are few studies looking at the feasibility of this procedure and the outcomes in an Asian population. This study aims to look at outcomes at our hospital.

Methods: We identified 121 patients treated with APBI at our center between 2008 and 2014 The median follow-up for our patient group was 30 months (range 3.7-66.5). The prescribed dose per fraction was $3.4 \mathrm{~Gy}$ in 10 fractions. In this study population, $71 \%$ of the patients were Chinese while $15 \%(n=19)$ were of other Asian ethnicity.

Results: In this study, the median breast volume was $850 \mathrm{cc}$ (range 216-2,108) with 59.5\% $(\mathrm{n}=72)$ patients with a breast volume of $<1,000 \mathrm{cc}$. The average planning target volume was $134 \mathrm{cc}$ (range 28-324). The number of catheters used ranged from 8 to 25 with an average of 18 catheters used per patient. We achieved an average dose homogeneity index of 0.76 in our patients. The average D90(\%) was 105\% and the average D90(Gy) was 3.6 Gy per fraction. The median volume receiving 100\% of the prescribed dose (V100) was $161.7 \mathrm{cc}$ (range 33.9-330.1), $150 \%$ of the prescribed dose (V150) and $200 \%$ of the prescribed dose (V200) was $39.4 \mathrm{cc}$ (range 14.6-69.6) and 14.72 cc (range 6.48-22.25), respectively. Our dosimetric outcomes were excellent even in patients with breast volume under $1,000 \mathrm{cc}$. There were no cases of grade 3 skin toxicity or acute pneumonitis. Two patients had a postoperative infection and two patients had fat necrosis postprocedure.

Conclusion: Multicatheter high dose rate APBI is a safe and feasible procedure that can be carried out with minimal toxicity in Asian patients with breast volumes under 1,000 cc.

Keywords: breast, Asian, multicatheter, brachytherapy

\section{Introduction}

Breast-conserving surgery followed by whole breast irradiation (WBI) has become the standard of care for early breast carcinoma. ${ }^{1-4}$ However, the need for WBI has been questioned and several centers have evaluated the feasibility and efficacy of accelerated partial breast irradiation (APBI)..$^{5-7}$ APBI represents a technique that allows for the delivery of adjuvant therapy after breast-conserving surgery, in 1 week or less, with multiple techniques being available at this time. To date at least five Phase III trials comparing different techniques of APBI to conventional WBI have been initiated. ${ }^{8-12}$ Polgár et al $^{13}$ demonstrated excellent long-term local tumor control, survival, and cosmetic results with a low-rate of long-term toxicity in a 12-year randomized prospective study using multicatheter interstitial brachytherapy. In the US, the Pooled Registry of Multicatheter Interstitial Sites study group also showed excellent long-term local
Correspondence: Johann I-Hsiung Tang Department of Radiation Oncology, National University Cancer Institute Singapore, IE Kent Ridge Road,

II 9228 Singapore

Tel +65 67724869

Email johann_tang@nuhs.edu.sg 
control and cosmesis in 1,356 patients who underwent multicatheter APBI. ${ }^{14}$ These studies have encouraged many centers around the world to explore and to set up APBI programs. However, most studies have been done in Europe and in the US. Little is known about the cosmesis and the feasibility of this procedure in Asian women. There is also concern among radiation oncologists that the procedure may be technically difficult in patients with smaller breast volume. Due to the smaller build of Asian women, many of our patients have breast volume of $<1,000 \mathrm{cc} .{ }^{15}$ Otani et $\mathrm{al}^{16}$ had previously found the use of multicatheter APBI in Japanese women with A and B cup-sized breasts feasible. However, breast volume was not mentioned in the study and quality assurance and plan assessment in Otani's study differed from other studies. The aim of this study was to present our findings in relation to a more quantifiable breast volume and to use the Radiation Therapy Oncology Group (RTOG) plan assessment to more accurately assess the feasibility of this technique. In this retrospective study, we report the dosimetric and toxicity outcomes of multicatheter high dose rate (HDR) APBI in our Asian population with breast volumes under 1,000 cc.

\section{Methods}

Between November 2008 and August 2014, 121 patients treated with APBI with multicatheter HDR interstitial brachytherapy were included in the study. All patients underwent lumpectomy and axillary nodal evaluation either by sentinel node biopsy or axillary clearance. Clips were placed in the cavity postsurgery at the superior, inferior, anterior, posterior, and lateral aspects to help define the cavity for APBI. Patient selection criteria were as follows: 1) tumors of $<3 \mathrm{~cm}$ in size, 2) no lymph node involvement, 3) negative surgical margins, and 4) no multicentric disease or extensive intraductal component. The study protocol was reviewed and approved by the Domain Specific Research Board (DSRB number 2007/00368) and written informed consent was obtained from the patients.

\section{Implant technique}

Within 8 weeks of lumpectomy and axillary nodal evaluation, patients underwent an interstitial implant using the template method performed in the supine position. ${ }^{17,18}$ Once the cavity was localized, the ipsilateral breast area was surgically prepared under sterile conditions. To avoid injuring the underlying chest wall structures or causing a pneumothorax, the overlying breast was pinched and gently lifted off the chest wall before applying the template and securing it. Three to five anchoring needles were then placed in an asymmetric pattern usually involving C-12. C-12 was the grid coordinates of the template that corresponded to the center of the template. The purpose of the asymmetric pattern was to aid easy orientation of the template in reference to the patient's anatomy as well as to secure and prevent slipping of the template from the breast.

A computed tomography scan was then obtained with the anchoring catheters in situ. These images were reconstructed on the Oncentra planning system Version 4.3 (Elekta AB, Stockholm, Sweden).

\section{Planning technique}

The planning target volume (PTV) was formed by expanding 15-20 $\mathrm{mm}$ from the contrast enhanced tumor cavity and any surrounding surgical clips. The PTV-evaluation (PTV-Eval) was that formed by excluding the $5 \mathrm{~mm}$ skin rind as well as the underlying chest wall muscle layer. The skin was also contoured on the sagittal slice depicting the largest breast contour (usually the slice showing the nipple) and the most anterior chest wall surface was also contoured. The images were then reoriented in Oncentra to depict the "template view" to determine the location of the remaining catheters. Next an overlying photocopy of the template on a transparency was placed on the top of the screen and the "template view" template magnification was matched 1:1 to the overlaid template transparency. The corresponding anchoring needle positions were then marked on the overlaid transparency and the chest wall and PTV-Eval contour were outlined onto the transparency (Figure 1). Once done, the catheter placement for the remaining catheters was easily determined.

The final step required replacing the needles with polyethylene tubing with a hemispheric button at each end. Extra attention was given to make sure that the button on the connector side of the remote afterloader was flush to the skin. After each catheter was trimmed and numbered, an en face picture of the implant showing the catheter numbers with respect to the breast anatomy was obtained to aid in the reconstruction of the catheters. Computed tomography-based simulation of the patient was performed with the patient in the supine position and the images were then transferred to the Oncentra treatment planning system.

\section{Dosimetry}

A total of $34 \mathrm{~Gy}$ in ten fractions, two fractions per day, 3.4 Gy per fraction, separated by at least 6 hours, given on five treatment days was delivered via a 192Ir remote afterloader in the supine position. Target coverage (PTV-Eval) 


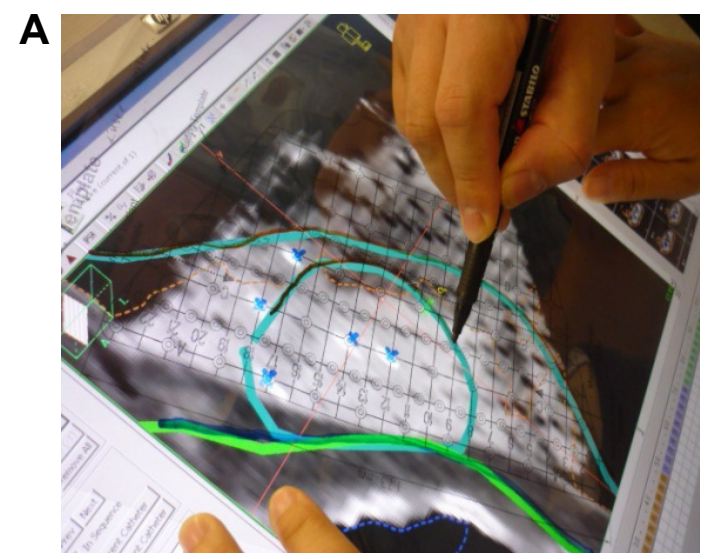

Figure I (A) Overlaying and tracing the contours onto the transparency template. placement.

was $\geq 90 \%$ of the prescribed dose covering $\geq 90 \%$ of the PTV-Eval (D90 $\geq 90 \%$ ). In our study, the dose constraint for the skin was set as $<100 \%$ of the prescribed dose. The skin was defined at the surface of the patient external contour. Care was taken to ensure that the $100 \%$ isodose line did not touch the patient contour. ${ }^{19}$

To ensure appropriate dose homogeneity throughout the implant, two parameters were used: the volume of tissue receiving higher doses and a dose homogeneity index (DHI). The actual volume of tissue receiving 150\% (V150) and $200 \%$ (V200) of the prescribed dose was limited to $\leq 70$ and $\leq 20 \mathrm{cc}$, respectively. The DHI, as represented by the volume ratio ( $1-\mathrm{V} 150 / \mathrm{V} 100$ ), was constrained to $\geq 0.75$. V150 represented the volume of tissue receiving $150 \%$ of the prescribed dose, and V100 represented the volume of tissue receiving the prescribed dose.

In addition, $<60 \%$ of the ipsilateral whole breast reference volume should receive $\geq 50 \%$ of the prescribed dose. The online Breast Atlas from the RTOG website was used to target the whole breast reference volume. ${ }^{20}$

\section{Toxicity}

Patients were reviewed at 1 week, then 3 months, and thereafter every 6 months. Patients were assessed for skin toxicity, such as radiodermatitis and telangiectasia, subcutaneous fibrosis, and fat necrosis. They were also assessed for symptomatic acute pneumonitis. These toxicities were graded using the Common Terminology Criteria for Adverse Events v3.0 where applicable. ${ }^{21}$

\section{Statistical analysis}

The results were analyzed statistically using the Stata v11.0 (Statacorp, College Station, TX, USA). The two-sample

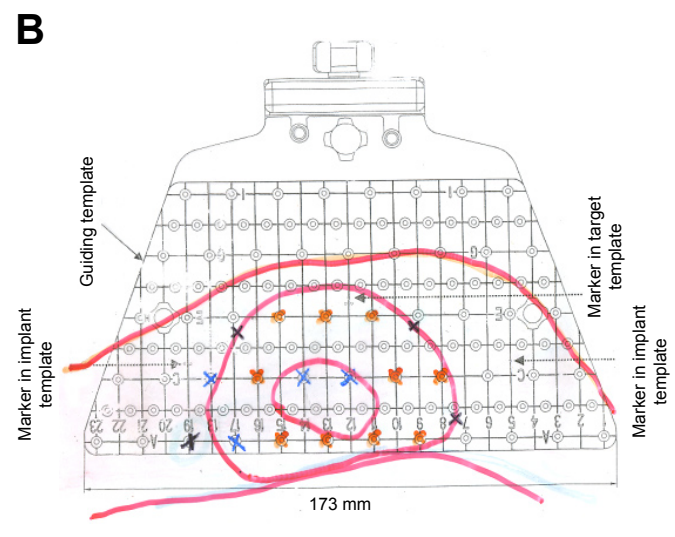

(B) Picture showing the transparency template with the positions of the catheter Wilcoxon rank sum (Mann-Whitney $U$-test) was used in the analysis given the small sample size.

\section{Results}

We identified 121 patients treated with APBI at our center between 2008 and 2014. The median follow-up for our patient group was 30 months (range 3.7-66.5).

\section{Patient and tumor characteristics}

In our study population, $71 \%$ of the patients were Chinese, while $15 \%(n=19)$ were of other Asian ethnicity. Ninety percent $(n=110)$ of our patients were at least 50 years old at the time of the implant. Eighty-two percent $(n=100)$ were postmenopausal (Table 1).

Seventy percent $(n=85)$ patients had T1 tumors, 12\% $(\mathrm{n}=15)$ had T2 tumors, and 17\% $(\mathrm{n}=21)$ had ductal carcinoma in situ. Most patients treated with APBI had estrogen receptor positive tumors (Table 2).

Table I Patient characteristics

Number of patients $(n=121)$

\begin{tabular}{ll}
\hline $\begin{array}{l}\text { Age at treatment (years) } \\
<40\end{array}$ & $5(4.1 \%)$ \\
$40-49$ & $16(13.2 \%)$ \\
$50-59$ & $63(52 \%)$ \\
$>60$ & $47(38.8 \%)$ \\
Ethnicity & \\
Chinese & $86(71.1 \%)$ \\
Indian & $4(3.3 \%)$ \\
Malay & $12(9.9 \%)$ \\
Others & $19(15.7 \%)$ \\
Premenopausal & $21(17.4 \%)$ \\
Median breast volume & $850 \mathrm{cc}($ range $216-2,108)$ \\
Breast volume under I,000 cc & $72(59.5 \%)$ \\
\hline
\end{tabular}


Table 2 Histological findings

\begin{tabular}{ll}
\hline & Number of patients $(\mathbf{n}=\mathbf{I} \mathbf{2} \mathbf{~})$ \\
\hline IDC & $22(18.2 \%)$ \\
GI & $33(27.3 \%)$ \\
G2 & $45(37.2 \%)$ \\
G3 & $21(17.4 \%)$ \\
DCIS & $9 \mathrm{~mm}(3-29)$ \\
Median tumor size & $87(71.9 \%)$ \\
Estrogen receptor positive & $20(16.5 \%)$ \\
LVI positive &
\end{tabular}

Abbreviations: DCIS, ductal carcinoma in situ; GI-G3, grade I-3; IDC, intraductal carcinoma; LVI, lymphovascular invasion.

\section{$\mathrm{APBI}$ in an Asian population}

In this study, the median breast volume was $850 \mathrm{cc}$ (range 216-2,108). The average lumpectomy cavity volume was $23.9 \mathrm{cc}$ (range 3.1-61.5). The average PTV was $134 \mathrm{cc}$ (range 28-324). The number of catheters used ranged from 8 to 25 with average of 18 catheters being used per patient.

We achieved an average DHI of 0.76 (range 0.57-0.84) in our patients. The average D90(\%) was $105 \%$ and D90(Gy) was 3.6 Gy per fraction. The median volume receiving $100 \%$ of the prescribed dose (V100) was 161.7 cc (range $33.9-330.1$ ), $150 \%$ of the prescribed dose (V150) and 200\% of the prescribed dose (V200) was $39.4 \mathrm{cc}$ (range 14.6-69.6) and $14.72 \mathrm{cc}$ (range 6.48-22.25), respectively. The maximum skin dose was 3.0 Gy per fraction (range 2.8-3.4).

We found $59.5 \%(n=72)$ patients with a breast volume of $<1,000 \mathrm{cc}$. In this group, the average number of catheters used was 16.7 and the average DHI and V100 was 0.74 and $134 \mathrm{cc}$, respectively. V150 was $38.5 \mathrm{cc}$ and V200 was $14.2 \mathrm{cc}$. There was no increased incidence of toxicity in this group of patients.

\section{Outcomes}

In our study, one patient relapsed with bone metastases and passed away two and a half years from the implant date. Due to the short follow-up, 26 patients were not due for their follow-up mammograms at the time of the data analysis. For the remaining patient cohort, there were no local recurrences detected on follow-ups.

\section{Postprocedure complications and toxicity}

There were two incidences of postoperative infection that resolved within a week with oral antibiotics. Two patients experienced fat necrosis that was diagnosed on routine ultrasound surveillance 36 months postimplant insertion. A summary of other toxicities observed in our study is shown in Table 3. No grade 3 toxicity was observed. Due to the low incidence of toxicity observed in our study group, we
Table 3 Incidence of toxicities

\begin{tabular}{ll}
\hline Toxicity & Incidence (number of patients) \\
\hline Telangiectasia & 1 \\
Radiodermatitis & 0 \\
Subcutaneous fibrosis & 3 \\
Fat necrosis & 2 \\
Symtomatic pneumonitis & 0 \\
\hline
\end{tabular}

could find no relationship between the breast volume and the incidence of toxicity observed.

\section{Discussion}

Our study shows that the multicatheter interstitial brachytherapy is dosimetrically feasible in the Asian population with good outcomes and with minimal acute toxicity. This is encouraging for centers in Asia that wish to start an APBI program.

Multicatheter interstitial breast brachytherapy has been gaining popularity as an adjuvant treatment for early-stage breast cancer due to its short treatment time, good outcomes, and excellent cosmesis.

As such, there is a need for sharing of expertise from various centers, especially in the use of this procedure in different ethnic populations. Studies documenting cosmesis and outcomes have been done in Europe and in the US. This is the first study that documents multicatheter interstitial APBI and the outcomes in an Asian population of largely Chinese ethnicity.

In the Asian population, it is fairly common to encounter breast volumes of $<1,000 \mathrm{cc}$, which can make APBI challenging. Yoshida et $\mathrm{al}^{22}$ reported the Japanese experience in APBI and cited challenges including small breast volume and high incidence of infection postoperatively. Small breast size were found to make insertion of the catheters and planning within the limits of the organs at risk difficult. Also, the use of open cavity technique, ${ }^{23}$ large V100, V150, and V200 volumes and nonuse of prophylactic antibiotics during the brachytherapy were found to be risk factors for infection. In our patients, we used the closed cavity technique and used prophylactic antibiotics throughout the brachytherapy, thus avoiding the problems of infection. We also ensured that the V100, V150, and V200 were within the RTOG technical guidelines thereby decreasing the chance of infection in our patients. Our results compare favorably with both Japanese and European series (Table 4).

In the Japanese studies, breast cup size was used as a determinant of breast size. Our study used breast volume to report on the outcomes. We found this to be more accurate than breast cup size reporting from the patient. Furthermore, the dosimetric findings in our study in patients with breast 
Table 4 Comparison of our results with other centers

\begin{tabular}{llll}
\hline Parameters & Singapore & Japan $^{22}$ & Hungary \\
\hline Local recurrence & $0 \%(30$-month follow-up) & $4 \%(3$ I-month follow-up) & 4.4\% (5-year follow-up) \\
Median DHI & 0.76 (range 0.57-0.84) & 0.7 (range 0.59-0.8) & I40.6 cc (range 38.5-3I5.I) \\
Median VI00 & I6I.7 cc (range 33.9-330.I) & I6\% wound complications \\
Toxicity & I.7\% wound complications & 50 cc (range II-82) & Not reported \\
\hline
\end{tabular}

Note: VI00 is the volume receiving $100 \%$ of the prescribed dose.

Abbreviation: $\mathrm{DHI}$, dose homogeneity index.

volume under 1,000 cc compared favorably with the current RTOG trial RTOG 04-13 technical guidelines for interstitial brachytherapy. This shows that multicatheter interstitial brachytherapy is a good treatment option for this subset of patients with good acute toxicity profile. Patients in this group have generally been considered technically challenging for interstitial brachytherapy. Our study shows that this procedure is very effective in patients with this breast volume while meeting the RTOG guidelines for treatment planning. The limitations of this study are the short follow-up. However, our study shares the experience of APBI in an Asian population with more than half of the subjects having a breast volume of $<1,000 \mathrm{cc}$. Future studies should focus on the long-term outcome of toxicity and recurrence in this study group.

\section{Conclusion}

Multicatheter HDR APBI is a dosimetrically feasible procedure that can be carried out with minimal acute toxicity in Asian patients with breast volumes under 1,000 cc.

\section{Disclosure}

The authors report no conflicts of interest in this work.

\section{References}

1. Litiere S, Werutsky G, Fentiman IS, et al. Breast conserving therapy versus mastectomy for stage I-II breast cancer: 20 year follow-up of the EORTC 10801 phase 3 randomised trial. Lancet Oncol. 2012;13(4): 412-419.

2. Fisher B, Anderson S, Bryant J, et al. Twenty-year follow-up of a randomized trial comparing total mastectomy, lumpectomy, and lumpectomy plus irradiation for the treatment of invasive breast cancer. NEngl J Med. 2002;347(16):1233-1241.

3. Veronesi U, Cascinelli N, Mariani L, et al. Twenty-year follow-up of a randomized study comparing breast-conserving surgery with radical mastectomy for early breast cancer. N Engl J Med. 2002;347(16):1227-1232.

4. Morrow M. Rational local therapy for breast cancer. NEngl J Med. 2002; 347(16):1270-1271.

5. Shah C, Antonucci JV, Wilkinson JB, et al. Twelve-year clinical outcomes and patterns of failure with accelerated partial breast irradiation versus whole-breast irradiation: results of a matched-pair analysis. Radiother Oncol. 2011;100(2):210-214.

6. Arthur DW, Winter K, Kuske RR, et al. A phase II trial of brachytherapy alone after lumpectomy for select breast cancer: tumor control and survival outcomes of RTOG 95-17. Int J Radiat Oncol Biol Phys. 2008;72(2): 467-473.

7. Benitez PR, Keisch ME, Vicini F, et al. Five-year results: the initial clinical trial of Mammosite balloon brachytherapy for partial breast irradiation in early stage breast cancer. Am J Surg. 2007;194(4):456-462.
8. Polgár C, Strnad V, Major T. Brachytherapy for partial breast irradiation: the European experience. Semin Radiat Oncol. 2005;15(2):116-122.

9. Vicini FA, Arthur DW. Breast brachytherapy: North American experience. Semin Radiat Oncol. 2005;15(2):108-115.

10. Polgár C, Major T. Current status and perspectives of brachytherapy for breast cancer. Int J Clin Oncol. 2009;14(1):7-24.

11. Gifford KA, Nelson CL, Kirsner SM, Kisling KD, Ballo MT, Bloom ES. On the feasibility of treating to a $1.5 \mathrm{~cm}$ PTV with a commercial singleentry hybrid applicator in APBI breast brachytherapy. J Contemp Brachytherapy. 2012;4(1):29-33.

12. Skowronek J, Wawrzyniak-Hojczyk M, Ambrochowicz K. Review article: Brachytherapy in accelerated partial breast irradiation (APBI) review of treatment methods. J Contemp Brachytherapy. 2012;4(3): 152-164.

13. Polgár C, Major T, Fodor J, et al. Accelerated partial-breast irradiation using high-dose-rate interstitial brachytherapy: 12-year update of a prospective clinical study. Radiother Oncol. 2010;94(3):274-279.

14. Kamrava M, Kuske RR, Anderson B, et al. Outcomes of breast cancer patients treated with accelerated partial breast irradiation via multicatheter interstitial brachytherapy: The Pooled Registry of Multicatheter Interstitial Sites (PROMIS) experience. Ann Surg Oncol. 2015;22(Suppl 3): S404-S411.

15. Li FY, He ZY, Xue M, Chen LX, Wu SG, Guan XX. Feasibility and acute toxicity of 3-dimensional conformal external-beam accelerated partial-breast irradiation for early-stage breast cancer after breastconserving surgery in Chinese female patients. Chin Med J (Engl). 2011; 124(9):1305-1309.

16. Otani Y, Nose T, Dokiya T, et al. A Japanese prospective multiinstitutional feasibility study on accelerated partial breast irradiation using interstitial brachytherapy: treatment planning and quality assurance. Radiat Oncol. 2015;10(1):126.

17. Tang JI, Tan PW, Koh YV, Buhari SA. Multi-catheter interstitial accelerated partial breast irradiation - tips and tricks for a good insertion. J Contemp Brachytherapy. 2014;6(1):85-90.

18. Koh YV, Buhari S, Tan PW, et al. Comparing a volume based template approach and ultrasound guided freehand approach in multicatheter interstitial accelerated partial breast irradiation. J Contemp Brachytherapy. 2014;6(2):173-177.

19. NSABP B-39: A randomized phase III study of conventional whole breast irradiation (WBI) versus partial breast irradiation (PBI) for women with Stage 0, I, or II breast cancer. Available from: http://www. rtog.org/ClinicalTrials/ProtocolTable/StudyDetails.aspx?study=0413. Accessed November 10, 2013.

20. RTOG Radiation Therapy Oncology Group. Breast Cancer Atlas. Available from: https://www.rtog.org/LinkClick.aspx?fileticket=vzJF hPaBipE\%3d\&tabid=236. Accessed November 10, 2013.

21. DCTD, NCI, NIH, DHHS. Cancer Therapy Evaluation Program. Common terminology criteria for adverse events, Version 3.0 (CTCAE); 2006. Available from: http://ctep.cancer.gov/protocolDevelopment/ electronic_applications/docs/ctcaev3.pdf. Accessed June 23, 2016.

22. Yoshida K, Nose T, Masuda N, et al. Preliminary result of accelerated partial breast irradiation after breast-conserving surgery. Breast Cancer. 2009;16(2):105-112.

23. Nose T, Komoike Y, Yoshida K, et al. A pilot study of wider use of accelerated partial breast irradiation: intraoperative margin-directed re-excision combined with sole high-dose-rate interstitial brachytherapy. Breast Cancer. 2006;13(3):289-299. 


\section{Publish your work in this journal}

OncoTargets and Therapy is an international, peer-reviewed, open access journal focusing on the pathological basis of all cancers, potential targets for therapy and treatment protocols employed to improve the management of cancer patients. The journal also focuses on the impact of management programs and new therapeutic agents and protocols on

patient perspectives such as quality of life, adherence and satisfaction. The manuscript management system is completely online and includes a very quick and fair peer-review system, which is all easy to use. Visit http://www.dovepress.com/testimonials.php to read real quotes from published authors.

Submit your manuscript here: http://www.dovepress.com/oncotargets-and-therapy-journal 